\title{
Trombo pedunculado ao nível da transição entre a aorta ascendente e o arco aórtico
}

\author{
Pedunculated thrombus at the transition of the ascending aorta to the aortic arch
}

João Carlos LEAL, Achilles ABELAIRA, Rita SANCHES, Domingo M. BRAILE

RBCCV 44205-673

\section{Resumo}

Homem, 52 anos de idade, alcoólatra, hipertenso, com história prévia de isquemia cerebral. Admitido no Departamento de Neurocirurgia com sinais e sintomas de acidente vascular cerebral isquêmico. A ecocardiografia transesofágica e tomografia computadorizada evidenciaram imagem sugestiva de trombo pedunculado ao nível da transição entre a aorta ascendente e o arco aórtico. $O$ tratamento de escolha foi procedimento cirúrgico com emprego da circulação extracorpórea associada a hipotermia profunda e parada circulatória total. Foi realizada ressecção do trombo e de parte parede da aorta para evitar reincidência. O paciente não apresentou intercorrências e teve alta hospitalar com uso de anticoagulante oral.

Descritores: Aorta, cirurgia. Aorta, patologia. Trombose. Trombectomia.

\begin{abstract}
A 52-year-old male alcoholic patient with hypertension and a prior history of brain ischemia was admitted to the neurosurgery department with signs and symptoms of ischemic stroke. A transesophageal echocardiogram and computed tomography suggested a possible pedunculated thrombus at the transition of the ascending aorta to the aortic arch. The therapeutic option was surgical treatment using cardiopulmonary bypass, deep hyperthermia and total cardiac arrest. The procedure involved resection of the aortic wall together with the thrombus to avoid recurrence. There were no adverse consequences and the patient was released from hospital with the use of oral anticoagulant drugs.
\end{abstract}

Descriptors: Aorta, surgery. Aorta, pathology. Thrombosis. Thrombectomy.
Braile Cardiocirurgia - São José do Rio Preto/SP

Endereço para correspondência: João Carlos Leal.Rua Luís de Camões, 3111 - Redentora.

São José do Rio Preto, SP, Brasil. CEP 15015-750 Fone (17) 2343555 .

E-mail: joaocarlos@braile.com.br
Artigo recebido em dezembro de 2003 Artigo aprovado em fevereiro de 2004 


\section{INTRODUÇÃO}

A presença de placa de ateroma presente na camada íntima da aorta possui um potencial desconhecido na formação de trombo pedunculado não obstrutivo, sendo este uma alteração rara, uma vez que as camadas da aorta podem ser normais.

Os fenômenos tromboembólicos nesta situação são complicações freqüentes. BARBON GARCIA et al. relataram oclusão da artéria retinal decorrente de trombo pedunculado localizado no arco aórtico [1]. Comprometimentos nas artérias de membros inferiores e coronárias podem apresentar conseqüências catastróficas para o paciente como insuficiência arterial periférica e infarto agudo do miocárdio (IAM), respectivamente [2,3].

Recentemente, RIBEIRO et al. publicaram artigo que descrevia o tratamento cirúrgico de um trombo pedunculado não oclusivo da aorta ascendente com história clínica prévia de acidente vascular cerebral isquêmico (AVCI) [4].

O objetivo deste relato de caso é demonstrar os riscos de eventos tromboembólicos extracardíacos.

\section{RELATO DOCASO}

Paciente masculino, 52 anos de idade, alcoólatra, hipertenso, três episódios de AVCI no período de quatro anos. Ao exame físico apresentava hemiplegia à direita, pulso diminuído em artéria femoral esquerda, dislalia e perda de campo visual bilateral.

A ecocardiografia transesofágica evidenciou imagem sugestiva de trombo pedunculado ao nível de transição entre a aorta ascendente e o arco aórtico de difícil visibilização devido ao distanciamento entre a aorta e o esôfago. A tomografia computadorizada confirmou o diagnóstico e foi possível mensurar a extensão da massa trombótica (Figura 1).

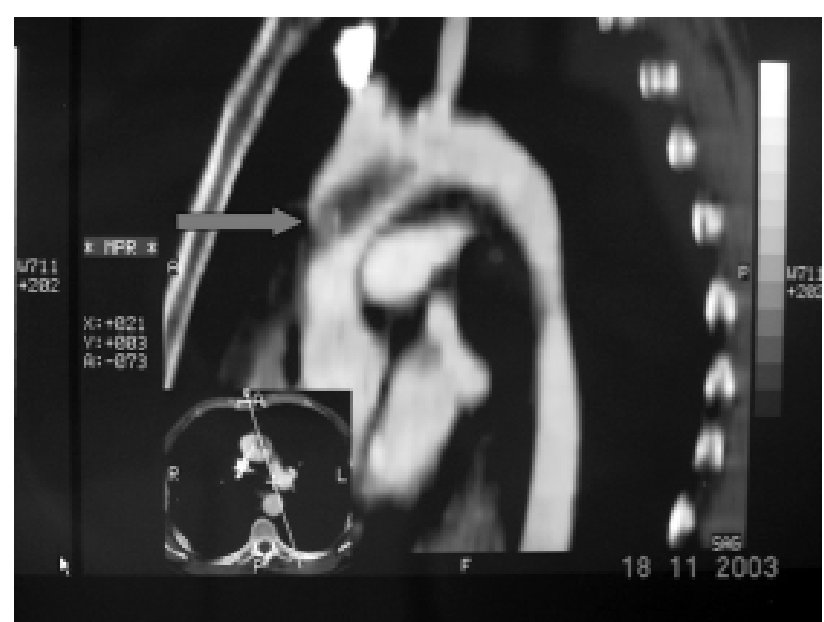

Fig. 1 - Tomografia computadorizada mostrando o trombo pedunculado na aorta ascendente (seta)
No procedimento operatório utilizou-se circulação extracorpórea (CEC) via artéria femoral associada a hipotermia profunda, parada circulatória total e microcardioplegia com proteção miocárdica. Abordagem da luz arterial foi realizada através da parede anterior da aorta ascendente com extensão da incisão até o terço médio do arco aórtico. Foi identificado e ressecado o trombo pedunculado móvel que estava aderido à parede anterior da aorta (Figura 2). Esta foi incisada circundando toda a origem do trombo e, em seguida, realizada a reconstrução da aorta com pericárdio bovino.

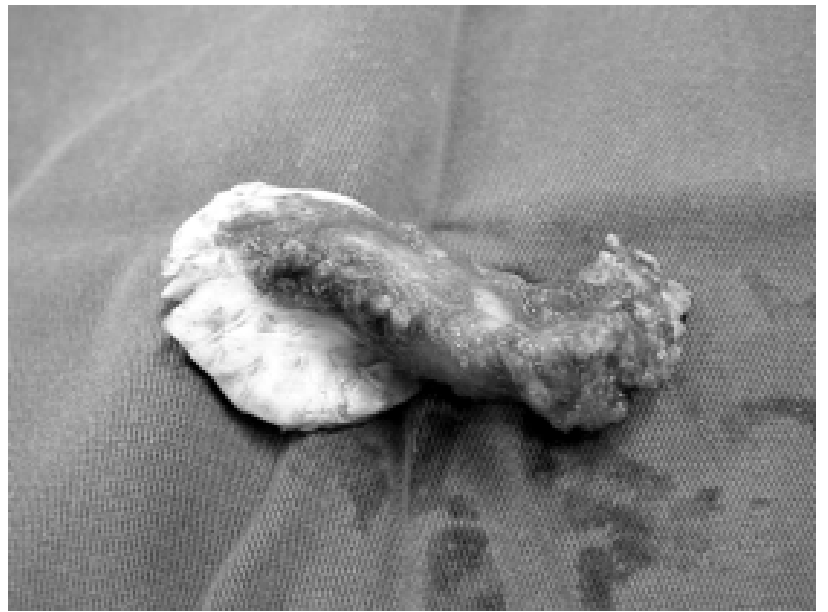

Fig. 2 - Ressecção de trombo pedunculado móvel aderido à parede da aorta ascendente

O local de aderência do trombo na aorta aparentemente apresentava uma placa de ateroma e a parede do vaso adjacente era de aspecto normal. $\mathrm{O}$ exame histopatológico macroscópico confirmou ser fragmento membranáceo, amarelado, firme-elástico, medindo 2,8 X 2,7 cm e em uma de suas faces centrada por formação polipóide, negra, friável, medindo 4,1 X 2,4 X 1,2 cm. A microscopia evidenciou fragmento de artéria elástica, com acentuadas alterações de aterosclerose nas túnicas íntima e média, sendo a primeira com a presença de área de erosão em continuidade com formação pediculada, constituída por faixas alternadas de hemáceas e fibrina.

No pós-operatório o paciente evoluiu sem intercorrências, recebendo alta hospitalar no sétimo dia com a recomendação de uso de anticoagulante oral.

\section{COMENTÁRIOS}

A presença de trombo pedunculado é rara em conduto arterial de alta pressão e fluxo, entretanto, suas complicações são graves decorrentes dos eventos tromboembólicos recorrentes ou não, marcados com seqüelas extracardíacas e/ou cardíacas [2]. 
A ecocardiografia transesofágica é o método diagnóstico de escolha para evidenciar o trombo pedunculado na aorta torácica e revelar lesão ulcerativa intimal mínima. Além disso, no ato cirúrgico serve como guia para o cirurgião [5]. Algumas dificuldades para visibilizar com nitidez a origem e a extensão da massa trombótica podem ocorrer. Para isto, deve-se realizar a ressonância magnética ou a tomografia computadorizada, principalmente nos casos em que o trombo origina-se na transição entre a aorta ascendente e o arco aórtico.

A heparinização intravenosa, seguida de anticoagulante oral, é uma alternativa terapêutica clínica indicada nos pacientes de alto risco para procedimento operatório. STOLLBERGER et al., em 2001, demonstraram absorção do trombo após 10 semanas e ausência de recorrência ao longo de 30 meses de seguimento [6].

A circulação extracorpórea associada a hipotermia profunda com parada circulatória total foi necessária para abordagem e avaliação do arco aórtico. É fundamental que a ressecção na parede da aorta adjacente ao trombo seja ampla, para evitar recorrência. Apesar das complicações serem freqüentes, graves e aparentarem uma doença de alto risco, a escolha do procedimento cirúrgico mostrou ser efetiva, eficaz e segura.

\section{REFERÊNCIAS BIBLIOGRÁFICAS}

1. Barbon Garcia JJ, Rodriguez Blanco V, Alvarez Suarez ML, Fernandez Lombardia M, Diez-Lage Sanchez A. A retinal embolism subsequent to atheroma in aortic arch. Arch Soc Esp Oftalmol 2001; 76:735-8

2. Lozano P, Gomez FT, Julia J, M-Rimbau E, Garcia F. Recurrent embolism caused by floating thrombus in the thoracic aorta. Ann Vasc Surg 1998; 12:609-11.

3. Bruno P, Massetti M, Babatasi G, Khayat A. Catastrophic consequences of a free floating thrombus in ascending aorta. Eur J Cardiothorac Surg 2001; 19:99-101.

4. Ribeiro PJF, Menardi AC, Vicente WVA, Évora PRB. Tratamento cirúrgico de um trombo pedunculado não oclusivo da aorta ascendente: apresentação de caso. Rev Bras Cir Cardiovasc 2003; 18:73-6.

5. Gabelmann M, Geibel A, Redecker M, Fraedrich G, Just H. The aortic arch as source of thromboembolism events: significance of echocardiography diagnosis. Z Kardiol 1995; 84:729-32.

6. Stollberger C, Kopsa W, Finsterer J. Resolution of an aortic thrombus under anticoagulant therapy. Eur J Cardiothorac Surg $2001 ; 20: 880-2$ 